\title{
LATOR: Protocolo de Roteamento Ciente de Qualidade com Prevenção de Aquecimento para Redes Corporais sem Fio
}

\author{
Egberto Caballero, Célio Albuquerque, Débora C. Muchaluat-Saade \\ Programa de Pós-Graduaçao em Computação. Instituto de Computação, \\ Universidade Federal Fluminense (UFF), Niterói - RJ - Brasil \\ \{egbertocr, celio, debora\}@midiacom.uff.br
}

\begin{abstract}
In this master thesis, the LATOR protocol was developed as a routing proposal for Wireless Body Area Networks (WBAN). LATOR aims to increase the Package Delivery Rate (PDR) in intra-WBAN communication and to prevent node overheating. LATOR considers the selection of routes based on the link quality information and prevents overheating by controlling node activity. The proposal was implemented and evaluated using the Castalia framework. Experimental results show that LATOR allows a better performance in the packet delivery rate when the direct communication is of low quality and there is some other alternative route with better quality. At the same time, overheating of the nodes in the network is avoided, maintaining an adequate PDR performance. LATOR slightly increases end-to-end latency; nevertheless, it is considered a viable alternative for WBAN applications.
\end{abstract}

Resumo. Nesta dissertação de mestrado foi desenvolvido o protocolo LATOR como proposta para o roteamento em Redes Corporais Sem Fio (WBAN). Este objetiva aumentar a Taxa de Entrega de Pacotes (PDR) na comunicação intraWBAN e prevenir o superaquecimento dos nós. Para isso, LATOR considera a seleção de rotas com base na informação de qualidade dos enlaces e evita o superaquecimento controlando a atividade dos nós. A proposta foi implementada e avaliada utilizando o framework Castalia. Resultados experimentais mostram que a proposta apresentada permite um melhor desempenho na taxa de entrega de pacotes quando a comunicação direta apresenta baixa qualidade e existe algum outro caminho alternativo com melhor qualidade comparado com a rota direta. Ao mesmo tempo se evita o superaquecimento dos nós na rede, mantendo um adequado desempenho no PDR. LATOR aumenta a latência fim-a-fim; porém, é considerado uma alternativa viável para aplicações em WBANs.

\section{Introdução}

Aplicações na área de saúde constituem a principal motivação para o desenvolvimento das Redes Corporais Sem Fio (WBAN), pois permitem fornecer monitoramento de sinais vitais em tempo real. No entanto, desafios devem ser superados para permitir sua adoção prática. Alguns desses desafios são: movimentos posturais, aquecimento dos nós, consumo energético, entre outros [Ferreira et al. 2018a]. Em WBANs, um aumento de temperatura dos nós de $0,1^{\circ} \mathrm{C}$ pode causar danos nos tecidos do corpo onde o nó é implantado [Caballero 2020]. O aquecimento dos tecidos na vizinhança dos nós implantados favorece a proliferação de bactérias e afeta o metabolismo das células. Por isso, o superaquecimento dos nós resulta em um desafio de alta importância a ser enfrentado. Também 
o problema de particionamento topológico devido ao movimento corporal e as baixas potências de transmissão é um desafio importante em WBANs. Em [Ferreira et al. 2018b] se mostrou que em cenários WBAN, nem sempre o caminho direto apresenta a melhor qualidade e que o uso de uma métrica de qualidade na seleção de rotas poderia melhorar o desempenho do PDR. Entretanto, na literatura não foi encontrada uma proposta para superar desafios como particionamento topológico e perdas de caminho baseada na qualidade do enlace, em um cenário de comunicação intra-WBAN. Por outro lado, no trabalho apresentado em [Zang and Li 2017], resultados experimentais mostram que existe uma relação direta entre o RSSI e a mobilidade do corpo humano. Como no padrão WBAN [Group et al. 2012] é definido o uso de topologia em estrela com comunicação direta entre os nós sensores e o nó coordenador, ou utilizando uma topologia de estrela estendida, usando no máximo dois saltos; trabalhos mais recentes estão focados em escolher um nó retransmissor; geralmente com base na energia residual e sem considerar a qualidade dos enlaces [Ferreira et al. 2018a].

Neste contexto, na dissertação [Caballero 2020] foi proposto um protocolo de roteamento para WBAN baseado na qualidade dos enlaces e com prevenção de superaquecimento dos nós. Este protocolo é denominado LATOR (Link-Quality Aware and Thermal Aware On-Demand Routing). O mesmo constitui a principal contribuição desta dissertação. LATOR consiste em um roteamento sob demanda, que explora a qualidade dos enlaces e a utiliza como métrica para a escolha das rotas. Para evitar o superaquecimento, se considera o calor gerado pela radiação das antenas e a potência dissipada pelos componentes eletrônicos dos nós. Entre os principais diferenciais do protocolo LATOR pode-se citar: (1) o aumento do PDR na comunicação intra-WBAN, quando a comunicação direta entre nós sensores e o nó coordenador tem baixa qualidade; (2) a seleção de rotas com base na informação de qualidade dos enlaces; (3) a mitigação do superaquecimento dos nós, sem trocar informação de temperatura entre nós vizinhos e; (4) a descoberta de novas rotas ante a quebra dos enlaces pelo movimento do corpo. Outra contribuição importante desta dissertação foi a implementação em Castalia de um módulo de temperatura que modela o comportamento da temperatura do nó e permite conhecer o estado de aquecimento do nó, quando desejado. Este módulo está baseado na equação de transferência de biocalor de Pennes e calcula a variação de temperatura com base na atividade do nó. Os módulos foram implementados em $\mathrm{C}++$. A avaliação foi realizada por simulação usando o framework Castalia no simulador OMNET++ e; utilizando parâmetros experimentais do comportamento do canal sem fio e considerando a mobilidade do corpo humano. Estas contribuições foram publicadas em 2 artigos principais: [Caballero et al. 2020a] e [Caballero et al. 2020b]. Também, no decorrer da pesquisa de dissertação ocorreram outras três publicações relacionadas a este trabalho, com a colaboração dos orientadores e colegas do grupo de pesquisa, as quais são [Ferreira et al. 2018a], [Lima et al. 2019a] e [Lima et al. 2019b].

\section{Protocolo LATOR}

O protocolo LATOR utiliza uma abordagem reativa para evitar muito tráfego de controle. Executa uma fase de descoberta de rota, onde são trocadas mensagens de Pedido de Rota (RREQ) e Resposta de Rota (RREP) e uma fase de manutenção, onde são trocadas mensagens Erro de Rota (RERR) e HELLO. Uma vez executada a descoberta de rota com sucesso, mensagens de dados (DATA) podem ser encaminhadas para o nó coordenador. 
A ideia geral da proposta consiste em coletar Informação da Qualidade dos Enlaces (LQI) durante a descoberta de rota. Depois os nós sensores usarão métricas baseadas no LQI para encaminhar os dados. A LQI que interessa a esta proposta é no sentido da comunicação sensor-coordenador, já que LATOR é projetado para melhorar o desempenho no sensoriamento. Simultaneamente cada nó verifica sua temperatura para tomar ações com o fim de evitar o superaquecimento. A prevenção do aquecimento se baseia no controle de atividade do nó. Cada nó compara sua própria temperatura com um máximo valor permitido a partir do qual considera-se que o nó está aquecido. Um nó aquecido entra em um estado de baixo consumo onde se descarta toda mensagem RREQ, RREP ou DATA recebida; as mensagens HELLO são processadas; quando são recebidas as mensagens RERR, as entradas afetadas são desativadas na Tabela de Rota (RT). Um nó sensor pode enviar mensagens DATA para o nó coordenador se não está aquecido. Caso contrário, é armazenado em um buffer até que a temperatura do nó alcance um valor abaixo do limiar definido. Se um nó intermediário entra no estado aquecido, envia uma mensagem RERR para todos os nós precursores de suas entradas na RT; notificando uma falha na rota.

A descoberta de rota é ativada quando um nó sensor fonte precisa enviar algum dado para o nó coordenador e não tem uma rota disponível na sua RT. Então, é transmitida uma mensagem RREQ em broadcast pela rede. Uma vez que as mensagens RREQs são descartadas pelo nó aquecido, a rota escolhida não tem nenhum nó aquecido. Para evitar loops na rede, cada nó sensor, que recebe o RREQ verifica se ele já recebeu anteriormente algum RREQ com o mesmo identificador. Se já foi recebido, o pacote é descartado; caso contrário o RREQ é processado. LATOR considera um número máximo de saltos para uma rota. Quando um nó recebe um RREQ incrementa o contador de saltos contido no RREQ. Se após ser incrementado, seu valor é menor que o número de saltos máximo permitido, a mensagem é processada; caso contrário é descartada. A coleta do LQI é realizada durante a disseminação do RREQ pela rede. Em LATOR, o nó coordenador responde todos os RREQs recebidos com um RREP, incluindo os duplicados de uma mesma solicitação. Os RREPs são enviados em unicast para o nó origem pelo caminho reverso. Enquanto o RREP percorre o caminho reverso, cada nó intermediário cria uma nova entrada para o nó coordenador indicando a lista de LQI da rota recebida no RREP. $\mathrm{Na}$ descoberta de rota, o nó sensor fonte deve esperar um tempo $T_{D}$ suficiente para receber todas as respostas enviadas pelo nó coordenador à mesma solicitação. No LATOR quando um nó detecta uma quebra de enlace devido ao movimento do corpo, notifica a todos os nós afetados enviando uma mensagem RERR para os nós precursores dessa rota. De forma similar, quando um nó intermediário alcança o estado de aquecimento envia uma mensagem RERR; mas nesse caso o RERR é enviado para os precursores de todas as entradas na RT do nó aquecido. Quando um nó recebe um RERR desativa na sua RT as entradas que têm como próximo salto o nó do qual recebeu o RERR. Então se não é o nó sensor fonte, ele encaminha o RERR para todos os seus precursores. O RERR não especifica se a falha é causada pela quebra de um enlace ou pelo aquecimento de um nó; mas esse procedimento garante que o nó origem conheça a falha na rota. Informações mais detalhadas do funcionamento e a implementação de LATOR estão disponíveis em [Caballero 2020]. 


\section{Avaliação e Resultados}

Na avaliação da proposta, foram considerados par fins de comparação: (1) o uso da comunicação direta, recomendado no padrão WBAN e (2) o protocolo "primeira rota" que modela o protocolo reativo AODV, (3) o protocolo LAOR (Link-Quality Aware On-Demand Routing), e (4) protocolo LATOR. Foi considerada uma topologia WBAN composta por um nó coordenador e cinco nós sensores; e considerada a mobilidade do corpo. As configurações usadas são válidas para sensores médicos de baixa taxa de transmissão, como temperatura, pressão arterial e saturação de oxigênio. Informações detalhadas do cenário simulado estão disponíveis em [Caballero 2020]. As simulações foram realizadas usando o framework Castalia e estão direcionadas em avaliar: a seleção de caminho com base no LQI e o controle de aquecimento. As métricas observadas foram o PDR, a latência da comunicação e aumento da temperatura. As simulações foram realizadas vinte vezes para todos os nós e os resultados mostrados são para o intervalo de confiança de $95 \%$.

No cenário utilizado na dissertação, o aumento de temperatura não atingiu o limite de $0,1^{\circ} \mathrm{C}$ referido na literatura. Então, para analisar o desempenho do método de prevenção do superaquecimento proposto, definiu-se um limite do aumento da temperatura de $0,0017^{\circ} \mathrm{C}$ nos nós sensores. No texto da dissertação são apresentados os resultados para todos os nós do cenário simulado. Neste resumo, só são apresentados os resultados correspondentes ao nó (3) da WBAN usada em [Caballero 2020]. Na Figura 1, é apresentado o comportamento da temperatura do nó 3 para este cenário. Pode-se ver na Figura 1(a) que com o protocolo LAOR o nó 4 experimenta um maior aumento na sua temperatura. Isso ocorre porque na maioria da vezes, ele atua como nó retransmissor do nó 3 , devido a que a rota 3-4-0 é a rota de melhor qualidade na maioria das vezes. Nos resultados apresentados na Figura 1(b), pode-se apreciar que com LATOR o aumento de temperatura não ultrapassa o limiar definido, evitando o superaquecimento dos nós da rede. $\mathrm{O}$ controle de temperatura é ativado pela primeira vez no segundo 2395 pelo nó 4; quando este alcança o estado de aquecimento. Os resultados mostram que utilizando a primeira rota, $28,30 \%$ dos pacotes transmitidos pelo nó 3 foram retransmitidos pelo nó 1 , quando este já tinha alcançado o estado de aquecimento. Com o protocolo LAOR, 36,45\% dos pacotes transmitidos pelo nó 3 foram retransmitidos pelo nó 4 no estado de aquecimento. Entretanto, o protocolo LATOR garante que não sejam feitas transmissões quando o nó está aquecido. Esses resultados mostram que o método proposto para o controle de temperatura funciona corretamente. Mas é importante analisar a influência desse método no desempenho de outros parâmetros como a latência e o PDR.

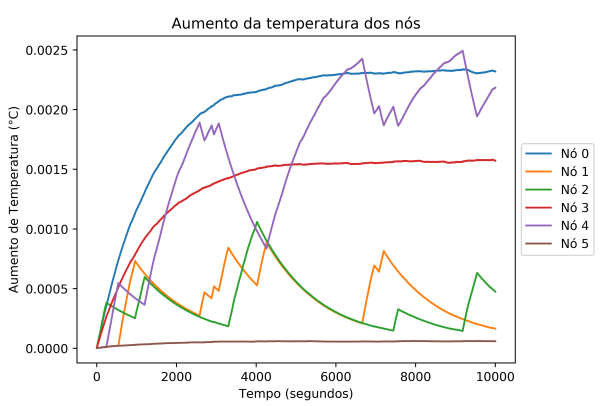

(a) LAOR, Nó 3 como transmissor

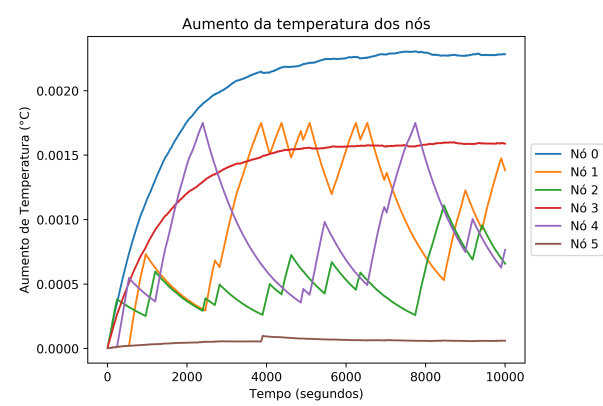

(b) LATOR, Nó 3 como transmissor

Figura 1. Comportamento da temperatura dos nós. 
Na Figura 2 é apresentada uma comparação do PDR. Os resultados mostraram que para o nó 3 , o enlace direto tem baixa qualidade, favorecendo a existência de rotas alternativas de melhor qualidade. Utilizando a rota direta, o nó 3 obtém um PDR de 67,53 $\pm 0,01 \%$. Usando a primeira rota encontrada se alcançou um PDR de 70,12 $\pm 1,03 \%$. Tanto LAOR quanto LATOR melhoram o PDR para o nó 3, alcançando um PDR de 74,89 $\pm 0,48 \%$ e $73,10 \pm 0,98 \%$ respectivamente. LAOR usou a rota $3-1-015 \%$ das vezes, a rota 3-2-0 16,81\% das vezes e a rota 3-4-0, de melhor qualidade, é usada $68,18 \%$ das vezes favorecendo o aumento do PDR. A troca de rota é realizada quando o nó 4 está aquecido e, fica temporariamente inabilitado para atuar como retransmissor. Então a rota 3-4-0 só é usada 33,55\% das vezes. Por isso, o PDR diminuiu comparado com LAOR, mas ainda oferece resultados melhores comparados com a rota direta e a primeira rota.

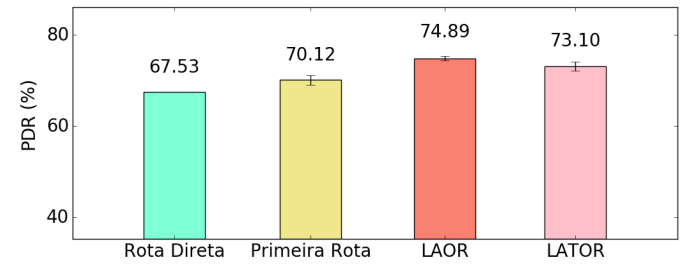

Figura 2. PDR quando o nó 3 atua como transmissor.

A latência fim-a-fim também foi analisada. A Figura 3 mostra o número de pacotes com uma dada latência. Na figura, é visível que quando se utiliza a rota direta há um maior número de pacotes com latência entre os primeiros $100 \mathrm{~ms}$. Para a primeira rota, LAOR e LATOR há um comportamento semelhante, aumentando a latência para alguns pacotes. Com a rota direta $0,8 \%$ dos pacotes recebidos têm uma latência maior que 500 ms; com a primeira rota encontrada 1,8\%; LAOR 2,02\% e LATOR 2,16\%. Esse aumento de latência corresponde principalmente à demora do processo de descoberta de rotas. Em compensação, aumenta a quantidade de pacotes que chegam ao nó coordenador.

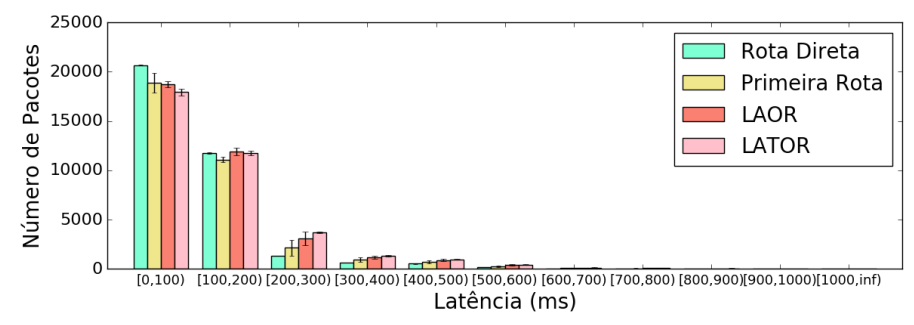

Figura 3. Distribuição da latência dos pacotes.

\section{Conclusões e Trabalhos Futuros}

Na dissertação de mestrado foi apresentado o protocolo LATOR, que propõe um mecanismo para a seleção de nós retransmissores baseados na qualidades dos enlaces e com prevenção do aquecimento dos nós. Também, foi desenvolvido um módulo de temperatura para aprimorar o framework Castaila. Este modela o comportamento da temperatura do nó e permite conhecer o estado de aquecimento do nó. Entre as principais contribuições do trabalho desta dissertação estão: o aumento do PDR na comunicação intra-WBAN, quando o enlace direto entre nós sensores e o nó coordenador tem baixa qualidade; a seleção de um nó retransmissor com base na qualidade dos enlaces; a mitigação do superaquecimento dos nós, sem trocar informação de temperatura entre nós vizinhos e; a escolha 
de um novo retransmissor ante a quebra dos enlaces pelo movimento do corpo. No decorrer da pesquisa de dissertação foram realizadas 5 publicações que apoiam a qualidade e a relevância da pesquisa para a comunidade científica da área [Caballero et al. 2020a], [Caballero et al. 2020b], [Ferreira et al. 2018a], [Lima et al. 2019a], [Lima et al. 2019b]. Como trabalhos futuros, é interessante valiar o comportamento da proposta com outros modelos de mobilidade do corpo ou em um testbed. Adicionalmente, considerar e avaliar o consumo energético é um aspecto importante para permitir um maior tempo de vida dos sensores.

\section{Agradecimentos}

Os autores agradecem a CAPES, o CNPq, a FAPERJ e a FAPESP pelo financiamento para desenvolver as atividades de pesquisa.

\section{Referências}

Caballero, E. (2020). Lator: Protocolo de roteamento ciente de qualidade com prevenção de aquecimento para redes corporais sem fio. In Dissertação de Mestrado, Instituto de Computação, Universidade Federal Fluminense.

Caballero, E., Ferreira, V., Lima, R., Albuquerque, C., and Muchaluat-Saade, D. C. (2020a). Lator: Protocolo de roteamento ciente de qualidade com prevenção de aquecimento para redes corporais sem fio. In Anais do XXXVIII Simpósio Brasileiro de Redes de Computadores e Sistemas Distribuídos, pages 854-867. SBC.

Caballero, E., Ferreira, V. C., Lima, R. A., Albuquerque, C., and Muchaluat-Saade, D. C. (2020b). Lator: Link-quality aware and thermal aware on-demand routing protocol for wban. In 2020 International Conference on Systems, Signals and Image Processing (IWSSIP), pages 337-342. IEEE.

Ferreira, V. C., Caballero, E., Lima, R., Balbi, H., Seixas, F. L., Albuquerque, C., and Muchaluat-Saade, D. C. (2018a). Redes corporais sem fio e suas aplicações em saúde. In Livro da 37a. Jornada de Atualização em Informática, pages 147-198. SBC.

Ferreira, V. C., Muchaluat-Saade, D. C., and de Albuquerque, C. V. (2018b). Estudo sobre estabilidade de rotas em redes corporais sem fio. In ERCAS-RJ 2018. SBC.

Group, I. . W. et al. (2012). IEEE Standard for Local and metropolitan area networks Part 15.6: Wireless Body Area Networks. Standard, IEEE, New York, USA.

Lima, R. A., Ferreira, V. C., Caballero, E., Albuquerque, C. V., and Muchaluat-Saade, D. (2019a). Uma proposta de camada de aplicação baseada no padrão ieee 11073 para simulação de aplicações de saúde digital em redes corporais sem fio. In Anais do XIX Simpósio Brasileiro de Computação Aplicada à Saúde, pages 234-245. SBC.

Lima, R. A., Ferreira, V. C., Caballero, E., Albuquerque, C. V., and Muchaluat Saade, D. C. (2019b). Simulation of iso/ieee 11073 personal health devices in wbans. In Proceedings of the 22nd International ACM Conference on Modeling, Analysis and Simulation of Wireless and Mobile Systems, pages 221-224.

Zang, W. and Li, Y. (2017). Gait-cycle-driven transmission power control scheme for a wireless body area network. IEEE journal of biomedical and health informatics, 22(3):697-706. 